\title{
Review: Principles of maximizing bull semen production at genetic centers
}

\author{
J. L. Schenk ${ }^{\dagger}$ \\ Schenk Animal Reproductive Solutions, LLC, 1906 Constitution Avenue, Fort Collins, CO 80526-1504, USA
}

(Received 24 October 2017; Accepted 15 February 2018; First published online 7 March 2018)

\begin{abstract}
Knowledge of the capabilities and limitations of the reproductive capacity of bulls is vital in maximizing reproductive efficiencies. Bull semen collection guidelines established by researchers and industry personnel to maximize the sperm harvest from bulls have been evolving for more than 60 years. Today, a mature artificial insemination industry employs those strategies to meet demands. These efficient management schemes exploit the reproductive potential of each sire while minimizing the associated risk of injury to bulls and reduce associated production costs. Personnel employed by a semen producing facility must be authorized to make effective and rational decisions based on principles of bull sexual behavior and reproductive physiology. Furthermore, collection facilities must be well planned to allow for the safe presentation of novel sexual situations while affording maximum safety for employees and proper footing for bulls. Normal bulls produce and ejaculate tremendous numbers of sperm. Most bulls have a sufficient libido for routine sexual activity, but become satiated to predictable stimulus situations. Frequent changes to the novelty should allow weekly harvest of four to six ejaculates per week for most bulls. Utilizing the physiological characteristics associated with each ejaculate to establish the collection frequency of each bull, and empowering an integrated collection and laboratory staff to monitor and make adjustments to the ejaculation frequency are necessary in maximizing the sperm harvest. Young bulls can ejaculate 10 to 20 billion sperm per week, and mature bulls should ejaculate 40 to 60 billion sperm per week. Semen collection management procedures should be reviewed when bulls do not meet production goals.
\end{abstract}

Keywords: bull, libido, novelty, semen, sperm

\section{Implications}

Semen collection personnel must possess a sound understanding of bull sexual behavior in order to maximize the sperm harvest to aide their organizations to compete in today's global marketplace. Testicular capacity, breed of a bull, age, physical ability, libido and body condition are important factors that should be independently evaluated when collecting semen from bulls. Use of novel sexual stimuli and false mounting bulls are mandatory to exploit the sperm harvest. Altering collection days and ejaculation frequencies based on sperm quality, estimated daily sperm production, and tabulated daily sperm output are necessary to meet established individual bull production goals.

\section{Introduction}

The bovine artificial insemination industry is again changing as young, high value genomic sires are collected, and semen is marketed globally. This genetic revolution is placing greater emphasis on bull semen production centers to maximize the

\footnotetext{
${ }^{\dagger}$ E-mail: schenkj|@yahoo.com
}

reproductive capacity of both young and adult bulls to meet global demands. Meeting these demands can be challenging, as perhaps more than half of the bulls presented for collection in today's market, may be less than 15 months of age. Furthermore, at any given time, a bull stud may experience difficulty obtaining sufficient high quality sperm from as many as $15 \%$ to $20 \%$ of bulls due to physical limitations or low libido. Production of ample product is a function of testicular capacity, daily sperm production (DSP) (Amann, 1970), daily sperm output (Amann et al., 1974), exploiting ejaculate potential in the laboratory, and timely bull management decisions.

The objective of any semen producing company is to maximize the sperm harvest while minimizing the risk of injury to bulls and employees. Taking advantage of the reproductive capacity of bulls is mandatory to satisfy market demands for artificial insemination organizations to have abundant product at a reasonable production cost to compete globally. Obviously, testicular size can be a limiting factor affecting sperm output, especially in younger bulls. Emphasis on the nutritional requirements for young bulls is essential for hastened development and future sperm producing abilities (Barth et al., 2008). With few exceptions, bulls with large testes 
produce more sperm than bulls with small testes (Almquist and Amann, 1961; Amann and Almquist, 1961). In general, older bulls have larger testes than young bulls. Concerns associated with bull breed, age, body condition, physical disabilities, nutritional requirements, libido, and environment can greatly influence sperm quality as well as sperm output. Unfortunately, there is not a one size fits all collection regime and ejaculation frequency that can be applied to all bulls all of the time. Bulls are individuals and must be managed as such, taking into account a multitude of considerations.

\section{Testicular assessment}

Maximal sperm harvest requires consideration of testis size. Scrotal measurements (Coulter and Foote, 1979) and assessments of testicular volume (Almquist and Amann, 1961) can be used to estimate testicular capacity. Scrotal circumference and paired testes weight have been shown to be highly correlated (Coulter and Foote, 1979). The size of the testes can be used to estimate DSP. Daily sperm production has been defined as the number of sperm produced per gram of testis, or per bull, per day. This estimate can be used to establish a collection frequency that maximizes sperm output while maintaining a high percentage of morphologically normal sperm.

Sperm are produced continually in the seminiferous tubules and then transported through the rete testis and efferent ducts to the epididymal duct. After puberty, the testis continues to grow rapidly as the seminiferous tubules become longer and their diameter increases as the lumen forms. Spermatogenesis becomes more efficient, thereby increasing the number of sperm produced daily per gram of testis. Maximum DSP per gram of testis is achieved about 20 weeks after puberty, although the testes continue to grow through 2 to 3 years of age (Almquist and Amann, 1976; Almquist, 1982). Other noteworthy seminal developments that occur $<10$ weeks post-puberty are the incremental improvements in the percentage of morphologically normal and motile sperm. These seminal characteristics are most likely associated with the normalization of spermatogenesis and epididymal function.

Weekly sperm harvest from 11 to 12-month-old bulls should approach 10 to 20 billion sperm, and for a 3 to 10-year-old bull, should be 40 to 60 billion sperm (Almquist and Amann, 1961; Almquist and Amann, 1976). Deviations from these documented production goals do occur, and are likely broader in a working production environment. Achieving a weekly production goal set for the testicular size of the bull in question is dependent upon his efficiency of sperm production (which cannot be controlled), the ejaculation frequency, and the ability of collection personnel to maximize the sperm harvest. Collection regimens not meeting the expected weekly sperm harvest or quality goals should be reviewed by management, barn and laboratory personnel.

\section{Epididymal development and function}

The epididymis provides a long duct through which all sperm produced by the testis are transported. Sperm are voided from this duct during ejaculation, masturbation or urination via the deferent duct and urethra. Anatomically, the epididymis is divided into three compartments. The caput, or head, functions to remove water and secrete compounds thought to be essential for sperm maturation (Cooper, 1986; Amann, 1987; Veeramachaneni et al., 1990). Maturation and acquisition of fertilizing capacity are completed in the corpus or body of the epididymis (Amann et al., 1974). The major area for maintenance and storage of fertile sperm is the cauda or tail of the epididymis. Age associated changes in epididymal storage capacity have been well documented (Almquist and Amann, 1961; Almquist and Amann, 1976). As caudal epididymal capacity increases through 2 years of age, so does DSP. The combined effect of these changes requires different collection regimens for young and mature bulls to maximize the sperm harvest. The larger the testes, the more sperm are produced and available for ejaculation. The tail of the epididymis of the bull has the ability to expand and contract to accommodate more or fewer sperm. It has been estimated that sexually rested, mature bulls have the ability to store 5 to 6 days' sperm production within the cauda (Amann and Almquist, 1961). For young bulls, an ejaculation frequency of one to two ejaculates on each of 3 non-consecutive days per week will maximize the weekly sperm harvest. For most mature bulls, collection of two to four ejaculates on each of 2 days per week will maximize the sperm harvest (Almquist, 1982).

If an appropriate semen collection regimen is implemented for each bull, the epididymal reserves can be depleted each collection day. Depleting the epididymal sperm reserves shrinks the size of the tail of the epididymis compared with that of a sexually inactive bull, shortening the transport time through the cauda by 1 to 2 days because there are fewer sperm contained therein. Therefore, a more youthful population of sperm is available for ejaculation (Amann et al., 1974). In addition, some storage induced sperm abnormalities (bent tails and detached heads) may be minimized when the epididymal sperm reserves are consistently depleted.

Ideally it is best to deplete epididymal sperm reserves on each collection day. This requires implementation of an integrated collection and laboratory team, whereby the ejaculation frequency is determined based on the physiological characteristics associated with each ejaculate. This united production staff philosophy to formulate an appropriate collection schedule for each bull holds employees accountable for the sperm harvest and subsequent exploitation of a bull's reproductive potential. It empowers employees with the freedom to make adjustments to the ejaculation frequency on a collection day basis rather than waiting for management to suggest and implement changes.

\section{Bull behavior and libido}

Understanding and recognizing bull sexual behavior indicators by semen collection personnel will enable the bull to ejaculate the most sperm per collection. Certainly there are libido differences among individual bulls that directly 
influence the ability to sexually arouse and prepare a bull for semen collection. In one study that compared the time to sexually stimulate beef bulls to that for dairy bulls. Researchers reported that it required nearly 10 times longer to stimulate beef $(10.9 \mathrm{~min})$ than dairy $(1.1 \mathrm{~min})$ bulls, based on time to the first mount, and almost three times longer to collect two successive ejaculates employing three false mounts from beef $(38.1 \mathrm{~min})$ than dairy $(12.6 \mathrm{~min})$ bulls (Almquist, 1973). Handling Zebu bulls differs for that of Bos taurus bulls. Turner (1980) reported that Zebu cattle are difficult to handle under forced or stressed working conditions. Therefore, working bulls that are easily stressed may require special environmental circumstances and extraordinary patience by the collection team. Semen collection should be an enjoyable event for the bull. Therefore, it is necessary to provide the bull with some essentials. Semen collection areas should be designed so that they provide excellent footing, incorporate adequate safety measures for both employees and bulls, and are void of distractions. Distractions such as tours, facility maintenance, feeding, and vehicle noises, etc. should be avoided. Bulls that are slow to respond are often easily distracted and can become nervous when non-routine activities interrupt the collection process. Some bulls are timid and reluctant to work near a dominant bull. Changing the bull line-up as well as the introduction time into the collection ring can sometimes be beneficial. Personnel must invest sufficient time over a series of consecutive collection days, observing a bull's mannerisms to develop a solid understanding of the bull's behavior to provide him with sexual situations that will entice him to become sexually stimulated.

Providing bulls with comfortable stalls that are heated or cooled for a temperate environment will positively impact sperm output, sperm quality, and bull well-being (Setchell, 2006). Sufficient bedding and/or rubber matting for stall flooring with help prevent and/or minimize foot, leg, back and spinal column discomforts. Excellent hoof condition is the most important physical aspect of pain-free semen collection. Lame bulls, whether from sole abscesses, foot rot, interdigital dermatitis, hoof cracks, laminitis, improper hoof trimming, etc. often will be hesitant to mount. Furthermore, lame bulls often overcompensate for the foot pain and exert abnormal pressure on other bone and muscle groups, placing the bull at risk for greater injury. Bulls must be physically sound on their feet when being asked to mount, dismount and thrust during the collection process. The use of complementary care veterinary treatments such as acupuncture and low-level laser therapy should be considered to ease pain discomfort when conventional methods are not proving useful.

\section{Sexual stimulation}

A collection frequency maximizing the harvest of epididymal sperm reserves must be incorporated for the highest obtainable morphologically normal ejaculate for any given bull. Bulls with large testes must be collected at a higher ejaculation frequency than less endowed bulls. Unfortunately, as collection frequency increases, reaction time and the need for novelty also increase. Reaction time is the period between the presentation of a bull to a stimulus animal and the first mount (Almquist and Hale, 1956). The degree of sexual pressure needed to elicit a sexual response varies among bulls. Some bulls can become sexually prepared in a relative short time period with limited novelty. Novelty is defined as providing a sexual stimulus that is unique and elicits a sexual response (Almquist and Hale, 1956). Novelty is deficient when a bull cannot routinely become sexually stimulated with the novelty presented to him within a reasonable time frame. Some bulls quickly become bored of routine sexual experiences. Novel approaches to evoke sexual stimulus that are unique and elicit a sexual response include: (a) presentation of the same teaser in a new location, (b) presentation of a different teaser, or (c) a combination of animals in the original location or in a new location. For a variety of safety precautions, mount animals should be castrated males. However, if safety procedures are observed, another bull may be utilized to elicit sexual behavior from the bull eligible for collection. Stimulus pressure is the number of novel approaches available for mounting and ejaculation per unit of time (Almquist and Hale, 1956). If the ejaculation frequency is increased, the stimulus pressure also must be increased to maintain a short reaction time. If a bull does not mount or show a response to a stimulus situation in $5 \mathrm{~min}$, a change in stimulus situation should be made. This is called the $5 \mathrm{~min}$ rule (Almquist and Hale, 1956). With adequate sexual stimulation, reaction time can be kept short. Alterations in bull sexual stimulation must be made in a timely manner to maintain the bull's interest, especially for bulls that are easily distracted. When the novelty presented to the bull is not working, something different should be tried.

Sexual preparation involves extending the interval of maintained stimulation beyond that required for mounting and ejaculation (Hale and Almquist, 1960; Hale, 1966). A bull not properly sexually prepared will have a few contractions of smooth muscles to move sperm to the distal portion of the cauda epididymidis, the deferent duct and/or pelvic urethra. The resulting ejaculate may contain a few sperm. Furthermore, the reaction time to first mount and successive mounts should be kept short. Sexual preparation achieved within the first 20 min could be lost if a bull loses interest as a result of prolonged sexual pressure due to his unwillingness to work. Bulls failing to ejaculate are likely inadequately prepared. A common mistake under this circumstance is to assume that the sample obtained is representative of the bull's reproductive abilities. Alternative means of collecting semen should be considered when a bull does not routinely produce what would be considered a 'normal' ejaculate following adequate sexual preparation procedures. Electro-ejaculation (Palmer, 2005) may assist in determining the bull's ability to produce and ejaculate sperm when faulty ejaculation is suspected. Furthermore, electro-ejaculation may be used as an alternate tool to achieve depletion of sperm reserves or for bulls that are reluctant and/or unable to become sexually prepared within a reasonable period of time. 
Novel ideas of sexual pressure to sexually stimulate and prepare bulls might include the following techniques. Bulls often become sexually aroused when watching mounting activity of other bulls. A common ploy is to position as many bulls around the collection ring as there is adequate room to safely manoeuver personnel and bulls. Bulls can be tied to the outside, or if there is sufficient space, within the collection ring. Bulls tied around the collection ring can watch other bulls mount. Alternatively, bulls can be placed into individual small pens facing the collection ring. This form of sexual stimulation usually will shorten the reaction time to ejaculation and contribute to the sexual preparation needed to maximize sperm output from all bulls. Bulls that are slow to work often are easier to sexually arouse when employing this technique.

Other useful tips in enticing bulls to mount include creating competition between them. Working two different bulls on the same mount and at the same time can create a competition between them. An uninterested bull could be mounted by another bull or teaser animal to elicit a sexual response. Rotating mount animals often after 5 or $10 \mathrm{~min}$ of active sexual stimulation will help maintain his interest in sexual activity. Collection personnel can employ multiple mounts when working bulls if needed. They can walk the mount animal(s) with the bull following in fast patterns (straight lines or circles), stopping abruptly at the first sign(s) of sexual interest. Perhaps they walk the mounts around the bull and allow the mount to rub against the bull in the process, taking sufficient time while maintaining the bull's interest. This manoeuver is completed by positioning the mount under the bull's chin. Collection personnel often coax the bull to mount with sounds of encouragement and/or by gently massaging his back and/or rubbing him between his rear legs. Should the bull become uninterested and listless, the collection staff might remove him from the active collection area and place him with a mount animal(s) in an isolated, darkened, very quiet area to avoid any distractions. If and when the bull begins to mount, it is important to let him continue to mount. Collection personnel should not discourage or distract a slow to work bull from mounting. They should also take note of the time of day a bull shows the most interest in sexual activity. Not all bulls want to have sex in the morning. Perhaps he will work better by collecting him in the afternoon. Herdsmen should be aware of any recent illnesses and immediately report their concerns to the staff veterinarian. In addition, veterinarians should be notified of any bull that cannot be sexually stimulated that otherwise has been routinely mounting, as this may be an indication of an illness. Collection staff might alter the feeding time to determine the time of day that is most conducive in getting a bull to work. They should avoid working bulls after a full feeding. It is the responsibility of barn personnel to make certain that bulls are housed in a comfortable environment with proper bedding. Timid bulls should not be housed near dominant, antagonistic, and/or vocal bulls as they can create a fearful environment for the shy bull.

\section{False mounts}

False mounting (Almquist and Hale, 1956; Almquist, 1973) is the most effective approach to sexual preparation, particularly because it is a clear indication of sexual arousal. It permits a bull to mount a teaser without eliciting the ejaculation response. A total of three false mounts followed by a fourth mount for semen collection will maximize the sperm harvest per ejaculate. The first two false mounts can occur in quick succession, followed by $2 \mathrm{~min}$ of active restraint (Almquist, 1973). Active restraint is the aggressive teasing of a bull without allowing mounting while maintaining sexual interest. Both false mounts and active restraint will increase the number of available sperm for ejaculation. Mounting opportunities can be limited for bulls having physical limitations or that suffer from low libido; 5 to $10 \mathrm{~min}$ of active restraint often can substitute for false mounting (Almquist, 1973), although it is unlikely that the sperm harvest will be maximized. For handicapped bulls with chronic and/or acute disabilities, collection personnel might consider reducing the number of ejaculates collected per day while increasing the number of collections days per week to achieve sperm harvest goals.

\section{Semen collection}

Semen collection is not a race against time. Each day, collection teams are assigned a group of bulls for semen collection. Usually it is advantageous to collect one ejaculate from each bull before attempting to collect the second ejaculate from any bull. This rotation should be repeated for the collection of successive ejaculates. This procedure provides each bull with an adequate refractory recovery period (Almquist and Hale, 1956; Hale and Almquist, 1960). A minimum of 20 to $30 \mathrm{~min}$ for most bulls is satisfactory. Each bull should be collected until the raw ejaculate concentration is $<800 \times 10^{6} \mathrm{sperm} / \mathrm{ml}$, and/or the ejaculate volume is $<1.0 \mathrm{ml}$. The number of ejaculates collected from a bull per day may increase or decrease, depending on the sperm concentration of the ejaculation. This collection approach will most often deplete the epididymal sperm reserves of a bull, maximizing the sperm harvest per collection day. Alternatively, a pre-determined number of ejaculates can be collected from a bull based on historical records of prior sperm output. It is unlikely that the epididymal sperm reserves of a bull will be consistently depleted using this collection strategy because an errant ejaculate containing a few sperm will get counted as a predetermined collection resulting in incomplete depletion of the epididymal sperm reserves.

\section{Maximizing the sperm harvest}

Procedures for maximization of sperm harvest have been well documented (Hale and Almquist, 1960; Almquist and Amann, 1976). Cognizance of the physiological characteristics associated with each ejaculate is needed to 
dictate the collection frequency of each bull. Ejaculate volume and concentration should be used to determine ejaculate frequency. Each ejaculate should be an acceptable representation of the ejaculate number (collection 1 to 4 during 1 day), collection frequency (collections/week) and testicular capacity of the bull. An integrated approach to semen collection involves laboratory and collection personnel to openly communicate with one another. A timely relay of seminal information is needed from laboratory personnel to collection personnel, enabling them to make individual bull sexual stimulus and preparation adjustments throughout a collection day that will help deplete the epididymal sperm reserves and maximize the sperm harvest. Laboratory technicians learn of extenuating circumstances about bull behavior or how the bull responded to the sexual preparation. Communication from collection personnel will help laboratory technicians better understand circumstances that influenced the ejaculate quality and quantity.

\section{Personnel}

Continued education of personnel in the field of harvesting bull sperm and sperm physiology is mandatory. Empowered employees get excited about what is possible only after they become educated and understand their roles in the production process (Lewis, 2015). Excited employees are often 'happy' employees and their positive attitudes will be reflected by the number of sperm harvested per bull per collection day. Collection personnel who recognize the need to increase sexual pressure and vary novelty should be encouraged to use their expertise. Refresher sessions reviewing the maximization of the sperm harvest should be performed periodically. Weekly and/or monthly production meetings analyzing production records for each bull are helpful to collection personnel. Too often, limited knowledge of bull reproductive physiology and lack of willingness to seek adequate sexual arousal of bulls limits the success of harvesting morphologically normal sperm. Personnel often believe they are attempting everything possible and achieving maximum sperm harvest; often, alternative procedures are left unexplored. Routine semen collection can result in personnel with 'tunnel vision.' Open discussion of bull sexual preparation, the use of novelty and different techniques is beneficial to collection personnel.

\section{Conclusion}

The current trend in the artificial insemination industry is to market semen from more and more young, genomic bulls. These bulls have limited sperm producing abilities due to their young age. This movement by the industry is likely to continue into the future. Therefore, it is mandatory to properly and actively manage the semen collection from bulls, but especially younger bulls. Most mature bulls can produce and ejaculate many more sperm than can be marketed. Too often, young bulls whose semen is in great demand fail to satisfy market needs in a timely manner. An aggressive approach to semen collection for finite periods followed by periods of sexual rest is the most efficient use of labor, minimizes routine sexual boredom, and results in higher quality sperm as opposed to limiting collections to a weekly event or limiting collection day ejaculates. Monitoring sperm output from each bull, addressing needs to properly sexually prepare bulls to maximize the sperm harvest, continual staff education, integrated production teams, and assessment of testicular capacity are essential for any artificial insemination organization to capitalize on the reproductive potential of genetically superior sires. Knowledge of sexual behavior of bulls, utilization of an array of stimulus situations, use of collection and ejaculation frequencies that assure maximal sperm harvest per ejaculate and a positive attitude are needed each and every day from collection personnel. Cohesive collection and processing teams, working in harmony during collection days place accountability on the employees to assure that ejaculate volume and concentration are properly represented. These valuable employees that monitor the collection process will assure the epididymal sperm reserves are adequately depleted and the sperm harvest is maximized for each bull each collection day.

\section{Acknowledgement}

Gratitude is extended to colleagues in industry and academia who provided insightful suggests for improving this manuscript.

\section{Declaration of interest}

None.

\section{Ethics statement}

This manuscript is an invited review of existing publications and as such did not use animals or statistics for its' preparation.

\section{Software and data repository resources}

None.

\section{References}

Almquist JO 1973. Effects of sexual preparation on sperm output, sperm characteristics and sexual activity of beef bulls with a comparison to dairy bulls. Journal of Animal Science 36, 331-336.

Almquist JO 1982. Effect of long term ejaculation at high frequencies on output of sperm, sexual behavior, and fertility of Holstein bulls; relation of reproductive capacity to high nutrient allowance. Journal of Dairy Science 65, 814-823.

Almquist JO and Amann RP 1961. Reproductive capacity of dairy bulls. II. Gonadal and extra-gonadal sperm reserves as determined by direct counts and depletion trials; dimensions and weight of genitalia. Journal of Animal Science 44, 1668-1678.

Almquist JO and Amann RP 1976. Reproductive capacity of dairy bulls. $\mathrm{XI}$. Puberal characteristics and postpuberal changes in production of semen and sexual activity of Holstein bulls ejaculated frequently. Journal of Dairy Science 59, 986-991.

Almquist JO and Hale EB 1956. An approach to the measurement of sexual behavior and semen production of dairy bulls. Proceedings, 3rd 


\section{Maximizing bull semen production}

International Congress on Animal Reproduction, Plenary Papers, Cambridge England, pp. 50-59.

Amann RP 1970. Sperm production rates. In The testis (volume I, ed. Johnson AD, Gomes WR and VanDemark NL), pp. 133-482. Academic Press, New York, NY, USA.

Amann RP 1987. Function of the epididymis in bulls and rams. Journal of Reproduction and Fertility, Supplement 34, 115-131.

Amann RP and Almquist JO 1961. Reproductive capacity of dairy bulls. V. Detection of testicular deficiencies and requirements for experimentally evaluating testis function from semen characteristics. Journal of Dairy Science 12, 2283-2291.

Amann RP, Kavanaugh JF, Griel LC Jr and VogImayr JK 1974. Sperm production of Holstein bulls determined from testicular spermatid reserves, after cannulation of rete testis or vas deferens, and by daily ejaculation. Journal of Dairy Science 57, 93-99.

Barth AD, Brito LF and Kastelic JP 2008. The effect of nutrition on sexual development of bulls. Theriogenology 70, 485-494.

Cooper TG 1986. The epididymis, sperm maturation and fertilisation. SpringerVerlag, Berlin, Germany.
Coulter GH and Foote RH 1979. Bovine testicular measurements as indicators of reproductive performance and their relationship to productive traits in cattle: a review. Theriogenology $11,297-311$.

Hale EB 1966. Visual stimuli and reproductive behavior in bulls. Journal of Dairy Science 25 (suppl. 1), 36-48.

Hale EB and Almquist JO 1960. Relation of sexual behavior to germ cell output in farm animals. Journal of Dairy Science 43 (suppl. 1), 145-169.

Lewis A 2015. Understanding ISO 9001:2015. Quality management system requirements. Environment for the operation of process. Clause 7.1.4, 179.

Palmer CW 2005. Welfare aspects of Theriogenology: investigating alternatives to electroejaculation of bulls. Theriogenology $64,469-479$.

Setchell BP 2006. The effects of heat on the testes of mammals. Animal Reproduction 4, 81-91.

Turner JW 1980. Genetic and biological aspects of Zebu adaptability. Journal of Animal Science 50, 1201-1205.

Veeramachaneni DNR, Amann RP and Palmer JS 1990. Proteins in luminal fluid of the ram excurrent ducts: changes in composition and evidence for differential endocytosis. Journal of Andrology 11, 140-154. 